Research Article

\title{
Esthetic Evaluation of Facial Soft Tissue Based on Nonrigid Image Deformation
}

\author{
Yali He $\mathbb{D}^{\text {, }}$, Yan Yang ${ }^{D}$, Abdelrahman Mohamed, and Genmiao Qi \\ Stomatology Department, Zhongnan Hospital, Wuhan University, Wuhan 430000, China \\ Correspondence should be addressed to Yan Yang; yangyan209@whu.edu.cn
}

Received 10 August 2020; Revised 14 September 2020; Accepted 20 October 2020; Published 3 November 2020

Academic Editor: Xiaojie Guo

Copyright ( ) 2020 Yali He et al. This is an open access article distributed under the Creative Commons Attribution License, which permits unrestricted use, distribution, and reproduction in any medium, provided the original work is properly cited.

\begin{abstract}
As the time passes by, orthodontics is paying more and more attention to facial aesthetics. However, related research is mostly in the stage of qualitative evaluation without clinical reference of specific soft tissue data. Therefore, by collecting the pre- and posttreatment photographs of 26 adult female patients, this study used image deformation technology to process the photos of a 23 year-old female patient, and two groups of facial photos were established. Then, 22 males and 27 females were selected to conduct an aesthetic evaluation to the original and processed photos by questionnaire survey. Relevant indicators of the corresponding photos were obtained. Group $t$-test, paired $t$-test, and nonparametric test were used in data calculation. For patients with high compliance, deep overbite with low angle or average angle are more suitable for fixed appliance with the bite plate, while with high angle are not suitable. The nonprofessionals prefer narrower face, more retracted lip position, and fuller chin than in the actual treatment. Among them, female evaluators prefer narrower lower face than male evaluators do. Male evaluators prefer thicker lips and chins, especially thicker lower lips than female evaluators do. Laypeople prefer narrower face, more retracted lip position, and fuller chin.
\end{abstract}

\section{Introduction}

With the rapid development of the Internet, the aesthetics has been influenced by online media. People have relatively higher requirements for their faces. According to reports, the pursuit of better facial aesthetics has become a very important reason for patients of orthodontic treatment [1-3]. Therefore, orthodontists are increasingly focusing on improving facial aesthetics when formulating treatment plans [4]. Patients' satisfaction is related to aesthetic perception [5], and different patients may have different views on facial aesthetics.

In previous studies, most of the evaluations on facial soft tissue aesthetics are in the qualitative stage, including Visualized Analoge Scale, Numerical Rating Scale (NRS), Verbal Rating Scale (VRS), Likert Scale, and Q-sort. Mary-Eleni et al. [6] rated the results of fixed orthodontic appliances and functional appliances by VAS, in order to study the impact of different treatment on improving the facial aesthetics of Class II patients. It is found that there is no significant difference in facial perception changes between the two treatment plans. Arqoub Sarah et al. [7] rated the 10 photos by NRS and drew conclusions about aesthetic evaluation related to gender and professional background. Havens et al. [8] found that the presence of malocclusion has negative impact on the overall aesthetics and orthodontic treatment can effectively improve the individual's facial coordination. McNamara et al. [9] analyzed the differences in the degree of interests of nonprofessionals in 6 facial regions (chin, ears, eyes, mouth, nose, and other regions) through eye tracking. Paiva et al. [10] evaluated 10 photos by the visual analog scale. They concluded that the vertical position of the maxillary canine teeth has an impact on the smile aesthetic evaluation, while gingival margin does not. Machado et al. [11] rated full-face photos and close-up photos by VAS to study the factors affecting the attractiveness of smiles. It is found that, in most cases, there is no statistical difference in aesthetics between orthodontists and laypersons. However, orthodontists are more strict with unilateral canine torque. Santos et al. [12] studied the impact of facial sagittal and vertical length on facial aesthetics by 
VAS. It is found that facial sagittal has a greater impact on facial aesthetics than the vertical height does. Pisulkar el at. [13] rated the photos with smiles with and without buccal corridor spaces by the visual analog scale. It is found that smiles with buccal corridor spaces are more attractive. Zange et al. [14] rated the photos with smiles with and without buccal corridor spaces by VAS and found that buccal corridor spaces have no impact on smile aesthetics. However, under the same degree of change in the buccal corridor, the short face type is more attractive than the long face type. Cuoghi et al. [15] rated and analyzed 71 photos of patients with long face by the Likert Scale and found that lack of lip seal and incisor exposition contributed most to unfavorable ratings. Filho et al. [16] selected 25 dentists (general clinicians and various specialties), 23 orthodontists, and 27 laypeople to evaluate smile and dental attractiveness through VAS and Q-sort. It is found that the accuracy of the two methods is similar. However, the latter is slightly more reliable than the former.

Visual analog scale is a $10 \mathrm{~cm}$ line segment. The starting point represents the least attractive, and the endpoint represents the most attractive. NRS and VRS are similar to VAS. The former ones convert the line segment into numbers, such as $0 \sim 10$, or corresponding figures, for evaluators to rate [7]. The latter establishes descriptive [17] text options according to certain logic for evaluators to rate. Likert Scale usually includes 5 or 7 options, and the evaluator selects the one that is the closest to personal aesthetics (for example, in 5 options' type, 1 score: very unpleasant, 2 scores: unpleasant, 3 scores: acceptable, 4 scores: pleasant, and 5 scores: very pleasant). $Q$-sort refers to the "forced normal distribution" method in the aesthetic evaluation of orthodontics. Evaluators are asked to put a specific quantity of photos in different items, such as the most attractive and least attractive. Define the boundary between attractive and unattractive until all the photos are rated, and then conduct the next relevant analysis.

Numerical Rating Scale standardizes the aesthetic feelings and improves the efficiency of clinical communication and plan formulation. However, the simple evaluation of feelings is influenced by many factors. There is a certain "ceiling effect" and it is still only a qualitative evaluation. Therefore, a new method is applied to quantitatively evaluate evaluation aesthetics in this study. Our challenge is to generate sets of data in the place of the data from the real treatment. This study processes the orthodontic photos of a 23-year-old female patient by the nonrigid image deformation method and established a set of frontal photos and lateral photos. Through the questionnaire, evaluators were required to select their preferred photos. The relevant indicators of the photos were measured. We can quantitatively compare the expectation with the actual clinical effect and differences between different groups of people.

\section{Methods}

2.1. The Frame of Quantitative Aesthetic Evaluation Method. As demonstrated in Figure 1, in this article, first we collected the pre- and post-treatment photographs of 26 adult female patients, used image deformation technology to process the photos of a 23-year-old female patient, and established two groups of facial photos (see Figures 2 and 3). Then, 22 males and 27 females were selected to conduct aesthetic evaluation to the original and processed photos by questionnaire survey. Finally, we measured photos and analyzed data (according to the research of scholars [18], select a 23-yearold female from the above orthodontic patients, which can exclude the influence of growth and aging changes on facial soft tissues).

2.2. Evaluation Metrics of Facial Soft Tissue. The subjects were 26 adult female patients between the ages of $18-35$ of the Department of Orthodontics, Zhongnan Hospital of Wuhan University, from 2017 to 2019, to exclude the impact of growth and gender on the study (see Figure 4).

The patients are divided into traditional fixed appliance with a bite plate and Invisalign group. There were 11 cases in the bite plate group and 15 cases in the Invisalign group. Selection and exclusion standards for patients: (1) patients of class; (2) with deep overbite more than II degrees; (3) patients with nonextraction treatment; (4) patients without dysplasia or periodontal diseases or TMD, and patients with the age $\geq 17$ years old who pass the growth peak; (5) patients with average angle or slightly low angle, excluding patients with obvious high angle; (5) patients with the first orthodontics; (6) exclude patients who have poor posture and expression in photos.

Canon EOS 90D was used to take photos of patients. The photos were saved in jpg format. Flip all the photos to the right. Make the line connecting glabella and pogonion of soft tissue perpendicular to the horizontal plane. Cut the photos, up to hairline, down to chin, front to pronasale, and back to ears. The first author used the same computer to measure in a continuous period of time. All photos were measured 3 times and every other week. The average value was obtained with no statistical difference.

Metrics: since direct data cannot be obtained from the photos, the ratio of each length to the interpupillary distance was used for comparison in this study. The marking points of frontal photos include pupil (P), zygomatic (Zy), gonion (Go), subnasal (Sn), and menton (Me) (see Figure 5 and Table 1). Photographing methods: sit properly, look directly at the camera, relax the lips, and keep mandibular postural position.

Marking points of lateral photos include (1) glabella (G), which refers to the foremost of glabella soft tissue; (2) nasion of soft tissue (Ns), which refers to the corresponding nasion on the lateral photos of soft tissue; (3) pronasale (Pn), which refers to the most anterior point of the tip of the soft tissue; (4) columella $(\mathrm{Cm})$, which refers to the most anterior point of the upper end of the columella; (5) subnasale (Sn), which refers to the connection point of the columella with the upper lip; (6) A-point of soft tissue (A'), which refers to the most concave point of the upper lip; (7) upper lip anterior (ULA), which refers to the most anterior point of the upper lip; (8) stomion (St), which refers to the foremost point where the upper and lower lips contact when the lips are 


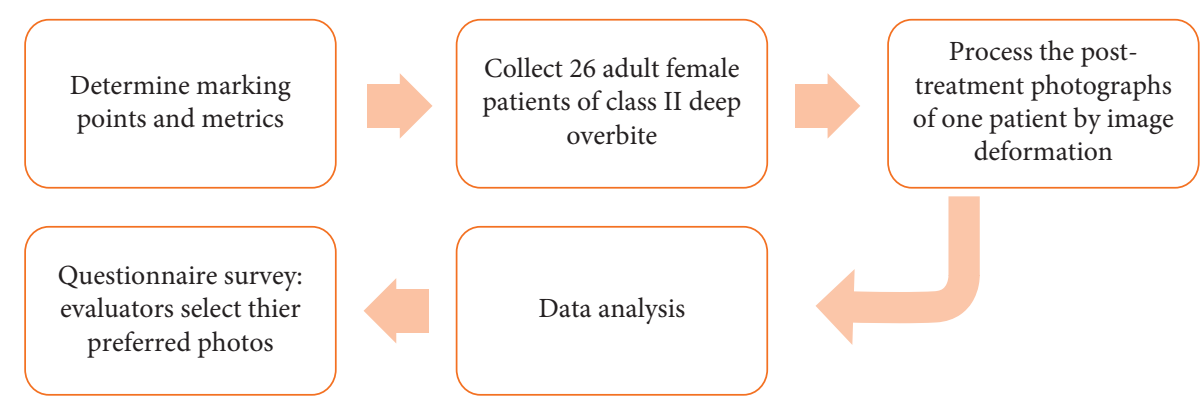

FIgURE 1: Flowchart of this article.

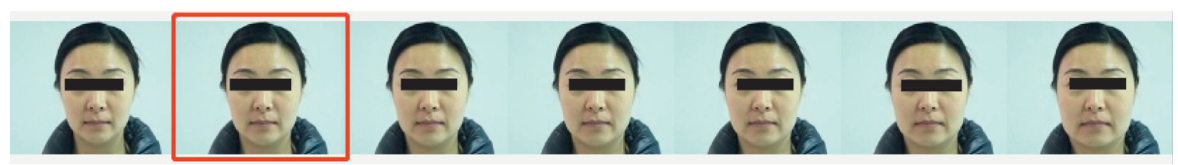

Figure 2: A set of frontal photos obtained by the image deformation method. From left to right, they are decreased grade III, decreased grade II, decreased grade (I) the original image, increased grade (I) increased grade II, and increased grade III. The red box indicates that the evaluator selected this item in the questionnaire.

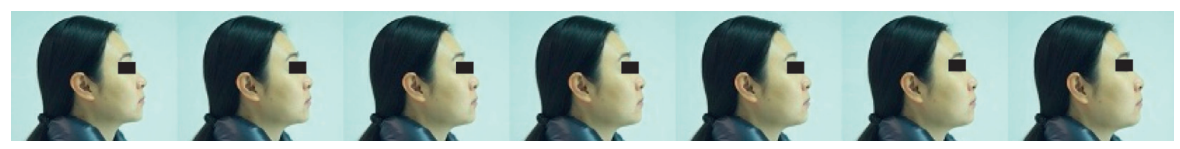

FIGURE 3: A set of lateral photos obtained by the image deformation method. From left to right, they are decreased grade III, decreased grade II, decreased grade (I) the original image, increased grade (I) increased grade II, and increased grade III.

naturally closed; (9) lower lip anterior (LLA), which refers to the most protruding point of the lower lip; (10) lower vermilion border (LV), which refers to the boundary point between the mucosa of the lower lip and the skin; (11) B-point of soft tissue (B'), which refers to the most concave point of the lower lip; (12) Pos, which refers to the most anterior point of soft tissue in the chin; (13) menton of soft tissue (Me'), which refers to the lowest point of the soft tissue in the chin; and (14) tragion (T), which refers to the upper edge of the cartilage of the tragion, where the base of the helix transitions to the skin. Reference plane: (1) OP plane, which refers to the connection between the midpoint of NsPos connection and $t$; (2) $E$ line, which refers to the tangent of Pn-Pos; and (3) $\mathrm{H}$ line, which refers to the connection between ULA and Pos (see Figure 6).

Measuring items: angle measurement indicators include G-Pn-Pos, G-Sn-Pos, Pn-Ns-Sn, Cm-Sn-ULA, OP-A'-ULA, OP-B'-LLA, Pos-B'-LV, ULA-Ns-Pos, OP-B'-LLA, LLA-NsPos, ULA-Ns-LLA, OP-Ns-Pos, and H-Ns-Pos (see Figure 6).

\subsection{Nonrigid Image Deformation Technology. Point-based} image deformation has developed for many years. Schaefer [19] proposed the Moving Least Squares (MLS) model to solve for image deformation, which constructs deformation function by linear functions such as rigid function and can generate well deformation results on rigid image deformation. Ma et al. [20] construct the displacement function by Gaussian kernel to solve the deformation function. Zhou
[21] solve for deformation function by TPS kernel; the model constructed by TPS has a free parameter. Both Ma et al. have generalized image deformation to nonrigid image deformation and can produce well deformation results with smoothness and natural [22]. For preserving more image details, Zhou introduces manifold regularization, which will be introduced later.

Qiu [23] proposed a nonrigid deformation method under the manifold constraint, which can generate natural and smoothness facial deformation result with intrinsic structure information preserved. The method solves deformation in a semisupervised and feature-guided fashion, which estimates deformation based on point handles, where they contain labeled deformation control point set and unlabeled feature control point set. Deformation control point set controls the deformation scales, and the feature control point set preserves the intrinsic geometric structure information of the source face.

Check area was defined with four distinct facial landmarks: the outer limit as the tragus; the inner border as the nasolabial groove; the upper limit to infraorbital margin and zygomatic arch; and the lower extended to the inferior region of the mandible; the front limit as a curve formed by subnascale, A-point of soft tissue, upper lip anterior, lower lip anterior, lower vermilion border, B-point of soft tissue, Pos, and menton of soft tissue. The landmark is obtained by a human-selected way, defined as the deformation control point. And the feature control points are the SIFT feature points extracted by VLFEAT [24] toolbox. Let $\left\{p_{i}\right\}_{i=1}^{N}$ and $\left\{q_{i}\right\}_{i=1}^{N}$ be the correspondence deformation control point 


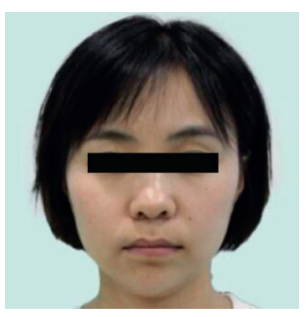

(a)

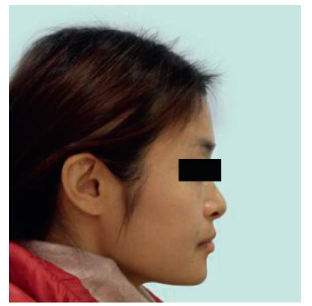

(f)

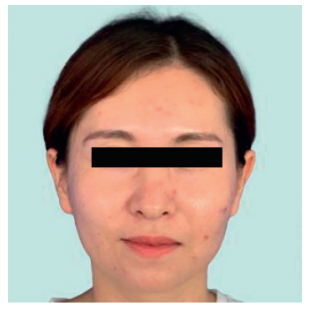

(k)

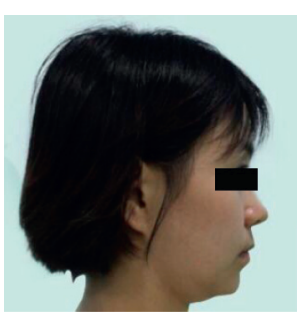

(b)

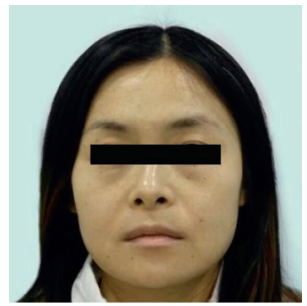

(g)

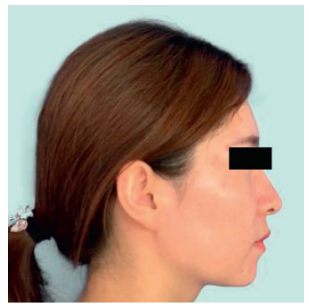

(l)

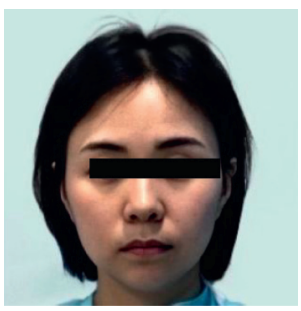

(c)

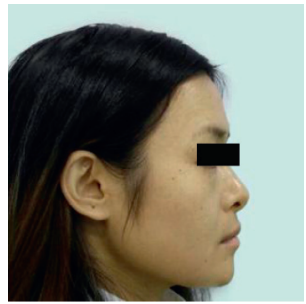

(h)

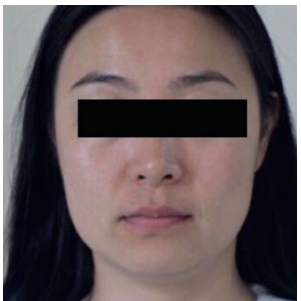

(m)

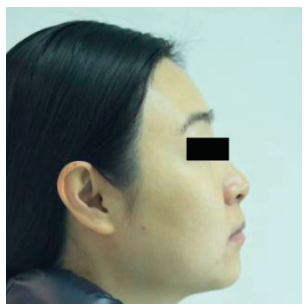

(p)

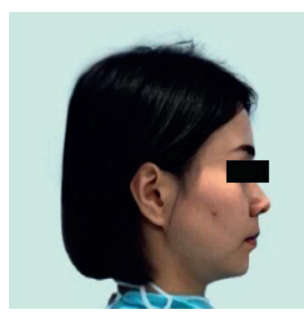

(d)

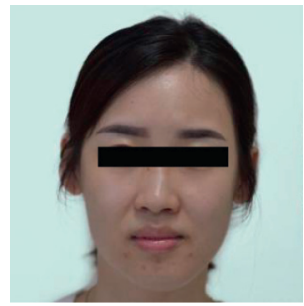

(i)

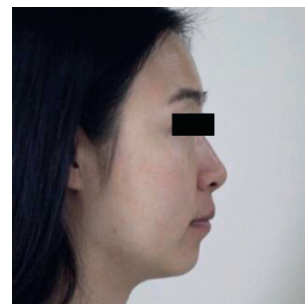

(n)

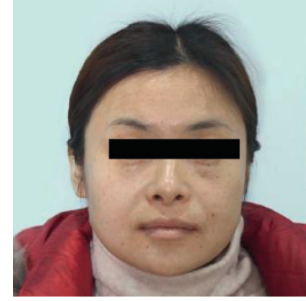

(e)

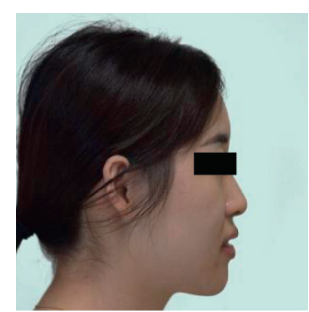

(j)

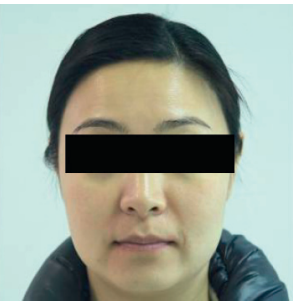

(o)

FIgURe 4: Photos from patients.

pair, $\left\{p_{i}\right\}_{i=N+1}^{M}$ be the feature control point, where $\left\{p_{i}\right\}_{i=1}^{M}$ is extracted from source face image and $\left\{q_{i}\right\}_{i=1}^{M}$ is extracted from the target face image.

Qiu [23] provided the deformation model based on traditional MLS (Moving Least Squares) method, which solves for rigid image deformation function by minizing the weight least squares error functional:

$$
\sum_{i=1}^{N} w_{i}(u)\left\|f_{u}\left(p_{i}\right)-q_{i}\right\|^{2},
$$

where $w_{i}(u)=\left\|u-p_{i}\right\|^{-2 \alpha}\left(w_{i}(u)\right.$ is nonnegative $)$ and $\alpha$ controls the weight of each control point. $\|\cdot\|$ denotes the Eucliden distance metric.

Owing to the traditional MLS, only take few deformation control points into account. And the method Zhou provided also takes feature control points to constraint the deformation; therefore, the model based on feature-guided moving least squares takes the following form:

$$
\sum_{i=1}^{N} w_{i}(u)\left\|f_{u}\left(p_{i}\right)-q_{i}\right\|^{2}+\lambda_{1} \varphi_{1}\left(f_{u}\right)+\lambda_{2} \varphi_{2}\left(f_{u}\right),
$$

where $\phi_{1}$ and $\phi_{2}$ represent the smooth regularization term and the manifold regularization term.

According to [23], deformation function is defined in a Reproduced Kernel Hilbert Space, which was constructed by the Gaussian kernel. The deformation was performed in the grid of the image, and deformation function of the arbitrary grid point $u$ was defined as follows:

$$
\begin{aligned}
f & =u+\sum_{i=1}^{M} \Gamma\left(\cdot, p_{i}\right) c_{i}, \\
V_{u}(\cdot) & =\sum_{i=1}^{M} \Gamma\left(\cdot, p_{i}\right) c_{i},
\end{aligned}
$$

with $\Gamma\left(\cdot, p_{i}\right)=e^{-\beta\left\|-p_{i}\right\|^{2}}$ and $c_{i}$ is the coefficient needed to be determined. $\beta$ determines the width of the range of 


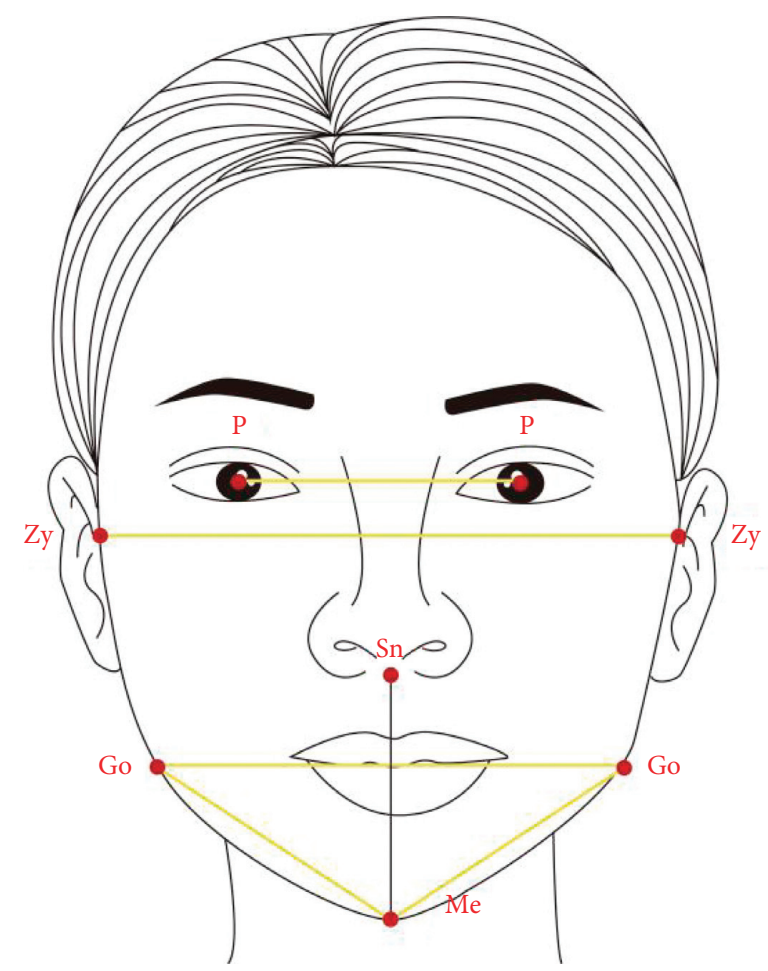

FIGURE 5: Marking points of frontal photos.

TABle 1: Metrics and meanings of P, Zy, Go, Sn, and Me.

\begin{tabular}{lcc}
\hline Measuring items & Metrics & Meanings \\
\hline The distanced of pupils & P-P & The distance between pupils, which is taken as reference \\
The width of zygomatic & Zy-Zy & The width of middle part of the face \\
The width of lower face & Go-Go & The width of the lower face \\
The height of lower face & Sn-Me & The height of the lower face \\
GMG angle & Go-Me-Go & The contour of the lower face \\
\hline
\end{tabular}

interaction. The nonrigid deformation function obtained by the minimized weighted regularized moving least square error functional:

$$
\sum_{i=1}^{M} \sum_{i=1}^{M} w_{i}(u)\left\|p_{i}+\Gamma_{u}\left(\cdot, p_{i}\right) c_{i}-q_{i}\right\|^{2}+\lambda_{1} \varphi_{1}\left(f_{u}\right)+\lambda_{2} \varphi_{2}\left(f_{u}\right) .
$$

The smooth regularization term can be defined as $\phi_{1}=$ $\left\|V_{u}\right\|_{\mathscr{H}}^{2}=\sum_{i=1}^{N} \sum_{j=1}^{M}<\Gamma\left(p_{i}, q_{i}\right) c_{i}, c_{j}>=\operatorname{tr}\left(\mathbf{C}^{T} \Gamma \mathbf{C}\right)$ with $\operatorname{tr}(\cdot)$ denoting the trace, where the kernel matrix $\Gamma \in \mathscr{R}^{\mathbf{M} \times \mathbf{M}}$ is Gram matrix and $C=\left(c_{1}, \ldots, c_{M}\right)$.

The manifold regularization term is defined by the graph Laplacian, which models a manifold by the weight neighborhood graph. And the weight neighborhood graph is constructed on the point set $\left\{p_{i}\right\}_{i=1}^{M}$, where $\left(p_{i}, p_{j}\right)$ is defined as edge only when $\left\|p_{i}-p_{j}\right\|^{2} \leq \epsilon$. The weight of the edge $\left(p_{i}, p_{j}\right)$ is defined as $G_{i j}=e^{(-1 / \epsilon)\left\|p_{i}-p_{j}\right\|^{2}}$. And the graph Laplacian matrix can be defined as $L_{i j}=\Lambda_{i j}-G_{i j}$ with $\Lambda_{\mathrm{ij}}=\operatorname{diag}\left(\sum_{i=1}^{M} G_{i j}\right)_{i=1}^{M}$. Therefore, the manifold regularization term defined as
$\phi_{2}\left(V_{u}\right)=\sum_{i=1}^{i=1} \sum_{j=1}^{M} G_{i j}\left(V_{i}-V_{j}\right)^{2}=\operatorname{tr}\left(\mathbf{V}^{T} L V\right)$ with $\quad \mathbf{V}=$ $\left(V_{u}\left(p_{1}\right), \ldots, V_{u}\left(p_{M}\right)\right)$.

To solve for the unknown coefficient matrix C, Zhou expresses the error functional in a matrix form:

$$
E(\mathbf{C})=\left\|\mathbf{W}^{1 / 2}(\mathbf{P}+\mathbf{J} \Gamma \mathbf{C}-\mathbf{Q})\right\|_{F}^{2}+\lambda_{1} \operatorname{tr}\left(\mathbf{C}^{T} \Gamma \mathbf{C}\right)+\lambda_{2} \operatorname{tr}\left(\mathbf{C}^{T} \Gamma \mathbf{L} \Gamma \mathbf{C}\right),
$$

where $\mathbf{W}$ is a diagonal weight matrix with $i$ th term being $w_{i}(p), \mathbf{P}$ and $\mathbf{Q}$ are composed of deformation control point set, $\mathbf{J}=\left(\mathbf{I}_{N \times N}, 0_{N \times(M-N)}\right)$ with 0 being a matrix composed of 0 , and $\|\cdot\|_{F}$ denotes the Frobenius norm.

Therefore, the closed form solution can be obtained:

$$
\mathbf{C}=\left(\mathbf{J}^{T} \mathbf{W} \mathbf{J} \Gamma+\lambda_{1} \mathbf{I}+\lambda_{2} \mathbf{L} \Gamma\right)^{-1}\left(\mathbf{J}^{T} \mathbf{W}(\mathbf{Q}-\mathbf{P})\right) .
$$

So, the expression of the deformation function can be written as follows:

$$
f(u)=u+\left[\Gamma_{u}\left(\mathbf{J}^{T} \mathbf{W} \mathbf{J} \Gamma+\lambda_{1} \mathbf{I}+\lambda_{2} \mathbf{L} \Gamma\right)^{-1}\left(\mathbf{J}^{T} \mathbf{W}(\mathbf{Q}-\mathbf{P})\right)\right]^{T}
$$




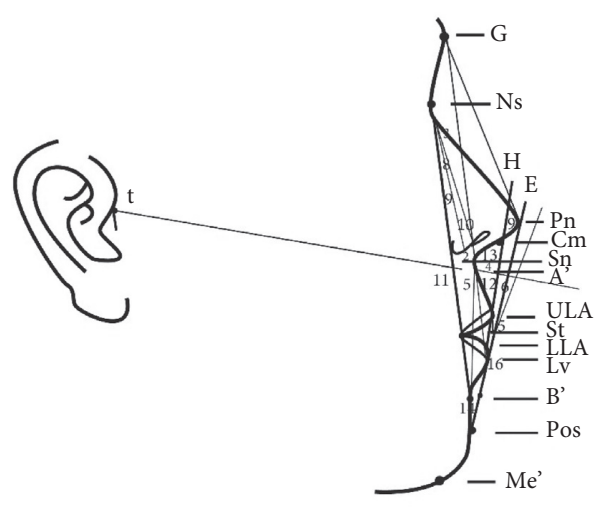

(a)

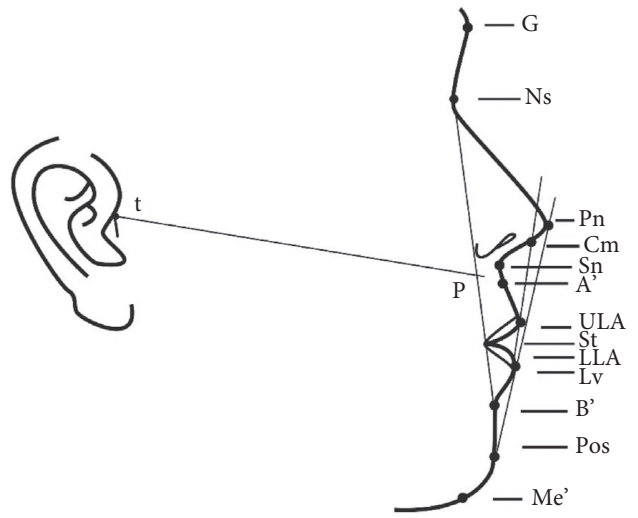

(b)

Figure 6: (a) Marking points and reference plane of lateral photos. (b) Metrics of lateral photos.

All the four parameters $\beta, \alpha, \lambda_{1}$, and $\lambda_{2}$ control the deformation result, where $\lambda_{1}$ and $\lambda_{2}$ control the smoothness regularization term and manifold regularization and $\alpha$ control the weight of each deformation control point.

After determining the original face image and the human-selected deformation control point, VLFEAT toolbox extracts the SIFT feature control point; then, we can obtain the deformation result by performing the deform model. We can obtain different deformation results by fine-tuning parameter $\alpha$ and $\beta$.

2.4. Questionnaire. The two sets of photos were submitted to 59 evaluators, including 22 males and 27 females. The criteria of evaluators: (1) Chinese nationality with no long-term overseas residence experience; (2) above 18 years old; (3) in good mental state; (4) nonprofessionals with no systematic orthodontics education. All evaluators were not related to the patients in the study.

The questionnaire firstly collected the basic information of evaluators, including gender, age, and occupation. After that, they were required to select their preferred photos. It is accompanied by an easy figure (see Tables 2 and 3 ).

The facial feature data preferred by evaluators is obtained through the same measuring method as the photos of the actual treatment.

2.5. Statistical Analysis. Most of the measurement data of this study conform to the normal distribution. The data is uniformly described by $(x \pm s)$. Paired-Sample $T$-test was used to compare the difference between the pre- and posttherapy to observe the clinical effects of the two treatment methods and Wilcoxon Signed Ranks test was used to analyze the nonnormal distribution data. Independent-Sample $T$-test was used to analyze the postoperative differences between two treatment methods and Mann-Whitney $U$ test was used when the data did not conform to the normal distribution. The data collected through the questionnaire did not conform to the normal distribution. Mann-Whitney $U$ test was used to compare the differences between actual
TABLE 2: Aesthetics of nonprofessionals.

\begin{tabular}{lccccccc}
\hline Front photo & $P 1$ & $P 2$ & $P 3$ & $P 4$ & $P 5$ & $P 6$ & $P 7$ \\
\hline Male & 9 & 6 & 1 & 1 & 4 & 1 & 0 \\
Female & 11 & 11 & 8 & 5 & 2 & 0 & 0 \\
\hline
\end{tabular}

TABLE 3: Aesthetics of nonprofessionals.

\begin{tabular}{lccccccc}
\hline Lateral photo & $P 1$ & $P 2$ & $P 3$ & $P 4$ & $P 5$ & $P 6$ & $P 7$ \\
\hline Male & 8 & 1 & 4 & 4 & 2 & 2 & 1 \\
Female & 17 & 10 & 3 & 4 & 2 & 1 & 0 \\
\hline
\end{tabular}

clinical effects and patients' expectations. The difference is statistically significant with $P<0.05$. In this study, SPSS (version 20.0; IBM, Armonk, NY, USA) was used for statistical analysis.

\section{Results}

3.1. The Comparison of the Two Treatment Methods. As demonstrated in Figure 7, through comparing the measuring data from post-treatment photos of the two orthodontic methods, the differences of two groups were statistically significant in the height changes of the lower face $(P<0.05)$. The patients of the fixed appliance with a bite plate group had an increase in the height of the lower face and narrowing of the lower face, while the Invisalign group did not show a significant increase in height.

\subsection{Differences of the Actual Clinical Effects and the Ideal} Aesthetic of Different Genders. In the actual effects of the fixed appliance bite plate and Invisalign group, only the zygomatic width/the distance between two pupils and the width/height of lower face were statistically different. Therefore, under the premise of considering these two metrics, the two groups can be combined into a group and then compared with the ideal aesthetics of different genders (see Figure 8). 


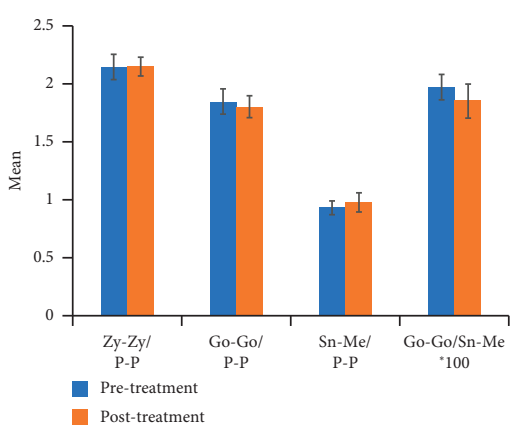

(a)

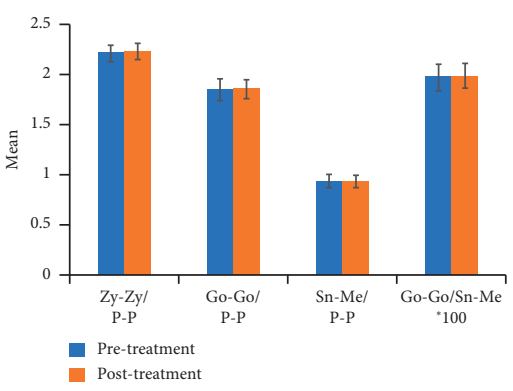

(d)

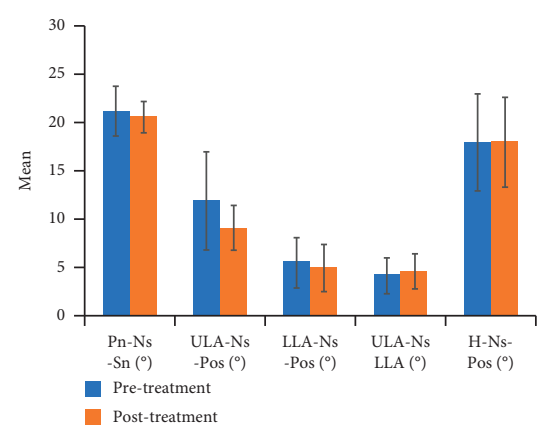

(b)

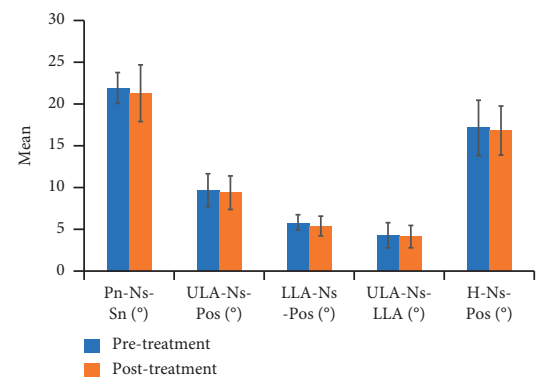

(e)

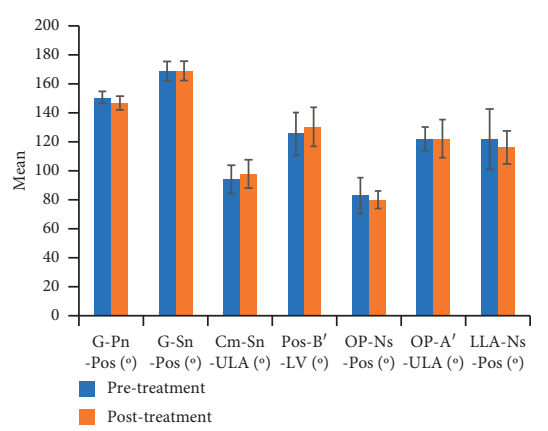

(c)

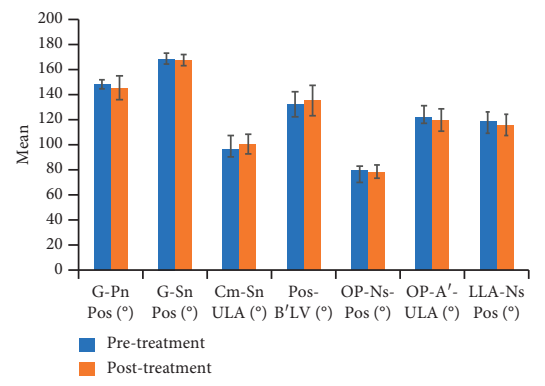

(f)

FIGURE 7: The comparison of the two treatment methods. (a)-(c) Bite plate group $(n=11)$, (d)-(f) Invisalign group ( $n=15) .{ }^{a}$ Wilcoxon signed ranks test.

The actual clinical effects are different from the aesthetics of different genders. Differences showed in cheekbone/the distance between two pupils, lower face height/the distance between two pupils, width/height of lower face, G-Sn-Pos, Cm-Sn-ULA, Pos-B'-LV, LLA-Ns-Pos, ULA-Ns-LLA, OPA'-ULA, OP-B'-LLA, and OP-Ns-Pos are statistically significant $(P<0.05)$. The cheekbone/pupil distance, lower height/pupil distance, G-Sn-Pos, Cm-Sn-ULA, ULA-NsLLA, OP-A'-ULA, and OP-Ns-Pos of the preferred photos of male and female evaluators are smaller than the actual clinical effects. Pos-B'-LV and LLA-Ns-Pos are smaller than the corresponding indicators of the actual clinical effects. It can be seen that both males and females prefer narrower lower face, more retracted lip position, and fuller chin than those of the actual treatment.

3.3. Aesthetic Differences of Different Genders. It is worth noting that the width/height of lower face and chin protrusion angle of the lower lip of the preferred faces of female evaluators are larger than male evaluators.

Female evaluators prefer a narrower lower face than male evaluators do. Male evaluators prefer thicker lips and chins, especially thicker lower lips than female evaluators do (see Table 4).

\section{Discussion}

The deep overbite of the anterior teeth is a common clinical abnormality in the vertical relationship. It is mainly caused by the excessive height of the anterior teeth or the insufficient height of the posterior teeth. The principle is mainly to depress the front teeth or prevent the front teeth from erupting, raise the posterior teeth, or both. Traditional fixed appliance with a movable bite plate is an effective method for deep overbite. It still allows some physiological adjustments to establish a more ideal occlusion [25]. The bite plate makes a certain occlusal gap for the posterior teeth, which is beneficial to the extension of the lower posterior teeth. However, compared with invisalign in terms of comfort and aesthetics, fixed appliance with a bite plate will reduce the compliance of patients. The biggest advantage of invisalign is aesthetics and comfort [26]. Besides, it controls the teeth from a three-dimensional direction and increases the control of twisting and tilting teeth. However, the occlusal surface of the invisalign will separate the cusp and the fossa of posterior teeth, which will make the teeth lose the opportunity for physiological adjustment. If worn for a long time, the invisalign may depress the posterior teeth like occlusal guards.

The results of this study showed that the lower face of bite plate group becomes higher and narrower, while the invisalign group had no obvious similar situation. This may be because that the deep overbite is not only on odontical factors but also on neuromuscular factors. The eruption of posterior teeth causes continuous tension on masseters. The clockwise rotation of the lower jaw also increases the tension of the buccal muscles. The posterior teeth do not contact with the bite plate, the mandibular condyle moves forward, and the movement of the mandible is free from the interference of the cusp. It is conducive to the normal development of the mandible and the growth of the alveolar height of the posterior teeth. At the same time, the muscle's anticontraction force effectively depresses the lower anterior 


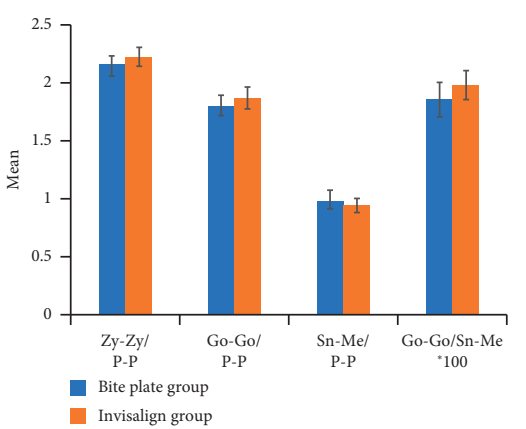

(a)

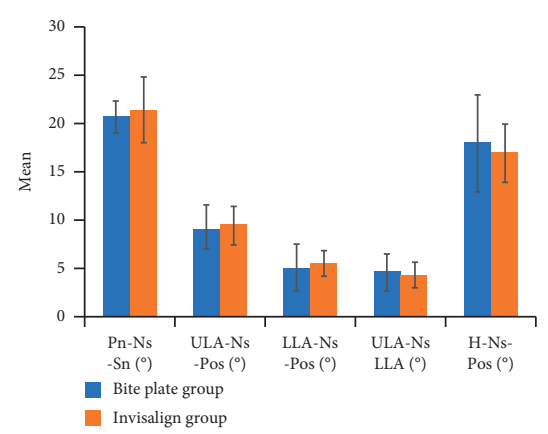

(b)

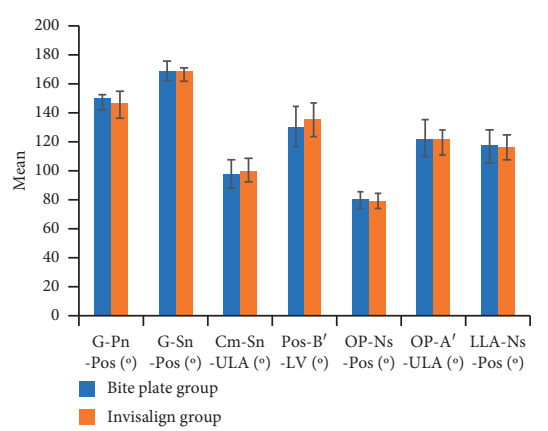

(c)

Figure 8: Post-treatment data of two treatment groups ( ${ }^{a}$ Mann-Whitney $U$ test).

Table 4: Differences of the actual clinical effects and the ideal aesthetic of different genders.

\begin{tabular}{|c|c|c|c|}
\hline Comparison $P$ (group $A-B)$ & Clinical groups-male evaluators & Clinical groups-female evaluators & Male evaluators-female evaluators \\
\hline $\mathrm{Zy}-\mathrm{Zy} / \mathrm{P}-\mathrm{P}$ & 0.027 & 0.015 & 0.603 \\
\hline Go-Go/P-P & 0.540 & 0.817 & 1.000 \\
\hline $\mathrm{Sn}-\mathrm{Me} / \mathrm{P}-\mathrm{P}$ & 0.002 & $<0.001$ & 0.770 \\
\hline Go-Go/Sn-Me $* 100$ & 0.112 & 0.037 & 0.042 \\
\hline G-Pn-Pos $\left({ }^{\circ}\right)$ & 0.135 & 0.166 & 0.244 \\
\hline G-Sn-Pos $\left({ }^{\circ}\right)$ & 0.022 & 0.041 & 0.076 \\
\hline Pn-Ns-Sn $\left({ }^{\circ}\right)$ & 0.089 & 0.091 & 0.358 \\
\hline Cm-Sn-ULA $\left({ }^{\circ}\right)$ & $<0.001$ & $<0.001$ & 0.544 \\
\hline Pos-B'-LV $\left({ }^{\circ}\right)$ & 0.006 & 0.003 & 0.566 \\
\hline ULA-Ns-Pos $\left({ }^{\circ}\right)$ & 0.740 & 0.611 & 0.341 \\
\hline LLA-Ns-Pos $\left({ }^{\circ}\right)$ & 0.023 & 0.004 & 0.024 \\
\hline ULA-Ns-LLA $\left({ }^{\circ}\right)$ & $<0.001$ & $<0.001$ & 0.301 \\
\hline OP-Ns-Pos $\left({ }^{\circ}\right)$ & 0.002 & 0.001 & 0.188 \\
\hline H-Ns-Pos $\left({ }^{\circ}\right)$ & 0.533 & 0.301 & 0.222 \\
\hline OP-A'-ULA $\left({ }^{\circ}\right)$ & 0.002 & 0.002 & 0.098 \\
\hline OP-B'-LLA $\left({ }^{\circ}\right)$ & 0.110 & 0.039 & 0.768 \\
\hline
\end{tabular}

teeth and relatively raise the posterior teeth. In the process of invisalign, the occlusal guards further increase the height of the posterior teeth area, lengthen the masseter fibers, and further depress the posterior teeth. Therefore, patients with high compliance and with deep overbite are more suitable for fixed appliance with a bite plate and patients' high angle is not suitable. Because the raise of the posterior teeth will further increase the height of lower face and make the mandible rotate clockwise. The face will become longer.

With the development of society and the progress of the times, more and more patients, especially adult female patients, pay more attention to whether the facial soft tissue is perfect. It is also an important goal of orthodontists [27]. In recent years, researchers in various countries have made many efforts to measure and define aesthetics. Since aesthetics is a subjective standard, it is difficult to establish clear aesthetic goals for clinical scheme [28]. Mary-Eleni [6] studied the facial aesthetic of orthodontic patients of Class II. Hata and Arai [29] studied smile aesthetics. They are both by VAS. However, the studies were only qualitative evaluation. In recent years, the widespread use of computer technology in the medical field has provided researchers with a new way of thinking. Therefore, this study proposed to use image deformation technology in aesthetic evaluation, to quantify different metrics, show people's aesthetics more objectively, explore whether the actual clinical effects meet laypeople's expectations, and guide the formulation of treatment scheme.

The results of this study show that the cheekbones/the distance between two pupils, lower face height/the distance between two pupils, G-Sn-Pos, Cm-Sn-ULA, ULA-Ns-LLA, OP-A'-ULA, and OP-Ns-Pos of the preferred faces of evaluators are less than the corresponding indicators in actual clinical effect. Pos-B'-LV and LLA-Ns-Pos are less than the corresponding indicators of the actual clinical effect. It can be seen that both males and females prefer a narrower lower face, more retracted lip position, and fuller chin than those of the actual clinical effect. This is consistent with the survey results of foreign scholars, such as Ioi [30] and Shimomura [31].

Also, the width/height of lower face and the LLA-Ns-Pos of the preferred photos of female evaluators were greater than the indicators of male evaluators. It is shown that female evaluators prefer a narrower lower face than male evaluators do. Male evaluators prefer thicker lips and chins, especially the former than female evaluators do. It can be seen that there are aesthetic differences between different genders, and between the actual clinical effects and the ideal aesthetic expectations of nonprofessionals. Therefore, both orthodontists and patients should fully communicate the 
ideal post-treatment facial soft tissues before. It helps to prevent the contradiction between orthodontists and patients due to aesthetic differences and excessive expectations.

This study also has deficiencies. This study only used the facial photos of a female volunteer. It did not analyze whether the orthodontic treatment has different effects on males. In addition, the skin color and facial features of the volunteer had an unavoidable effect on the evaluation results. In the future, a more in-depth study will be conducted by increasing the number of samples and the diversity of evaluators.

\section{Conclusions}

In this study, we can "sculpt" our favorite variation of a reference facial image through the image deformation method instead of rating the natural faces. It can be seen from the results of this study that the image deformation method is helpful to analyze the preferred faces of different people and the differences between the actual clinical effects and the ideal aesthetic expectations of the nonprofessionals.

Conclusions of this paper are drawn below:

(1) For patients with high compliance, deep overbite with low angle or average angle are more suitable for fixed appliance with a bite plate, while with high angle are not suitable.

(2) Female evaluators prefer a narrower lower face than male evaluators do. Male evaluators prefer fuller lips and chins, especially the former than female evaluators do.

(3) It can be seen that laypeople prefer a narrower face, more retracted lip position, and fuller chin.

\section{Data Availability}

The data used to support the findings of this study cannot be shared in order to protect patient privacy.

\section{Conflicts of Interest}

The authors declare that there are no conflicts of interest regarding the publication of this paper.

\section{References}

[1] J. E. Albino, R. N. Fox, E. A. Lewis, M. J. Slakter, and L. A. Tedesco, "Variables discriminating individuals who seek orthodontic treatment," Journal of Dental Research, vol. 60, no. 9, p. 1661, 1981.

[2] T. M. Cunat, "Factors influencing the desire for orthodontic treatment," European Journal of Orthodontics, vol. 3, pp. 151-162, 1981.

[3] L. A. Tedesco, "A dental-facial attractiveness scale: part I-reliability and validity," American Journal of Orthodontics, vol. 83, no. $1,1983$.

[4] R. M. A. Kiekens, "Objective measures as indicators for facial esthetics in white adolescents," The Angle Orthodontist, vol. 76, no. 4, pp. 551-556, 2006.

[5] C. Pachêco-Pereira, "Factors associated with patient and parent satisfaction after orthodontic treatment: a systematic review," American Journal of Orthodontics \& Dentofacial Orthopedics, vol. 148, no. 4, pp. 652-659, 2015.

[6] Z. Mary-Eleni, "Facial esthetic outcome of functional followed by fixed orthodontic treatment of class II division 1 patients," Progress in Orthodontics, vol. 20, no. 1, p. 42, 2019.

[7] H. Aroid Sarah and S. N. Al-Khateeb, "Perception of facial profile attractiveness of different antero-posterior and vertical proportions," European Journal of Orthodontics, vol. 1, pp. 103-111, 2011.

[8] D. C. Havens, L. M. Sigler, and T. Baccetti, "The role of the posed smile in overall facial esthetics," The Angle Orthodontist, vol. 80, no. 2, pp. 322-328, 2010.

[9] E. K. McNamara, F. M. Beck, A. R. Firestone, and S. F. Rosenstiel, "Role of facial attractiveness in patients with slight-to-borderline treatment need according to the aesthetic component of the index of orthodontic treatment need as judged by eye tracking," American Journal of Orthodontics and Dentofacial Orthopedics, vol. 151, no. 2, pp. 297-310, 2017.

[10] T. T. D. Paiva, A. T. Motta, and C. T. Mattos, "Influence of canine vertical position on smile esthetic perceptions by orthodontists and laypersons," American Journal of Orthodontics and Dentofacial Orthopedics, vol. 153, no. 3, p. 371, 2018.

[11] T. C. B. Machado and A. W. Machado, "Influence of maxillary canine torque variations on the perception of smile esthetics among orthodontists and laypersons," Dental Press Journal of Orthodontics, vol. 24, no. 1, pp. 53-61, 2019.

[12] K. Santos, "Association of facial sagittal and vertical characteristics with facial aesthetics in the northern Finland birth cohort 1966," The European Journal of Orthodontics, vol. 41, 2018.

[13] S. K. Pisulkar, "Perception of buccal corridor space on smile aesthetics among specialty dentist and layperson," Journal of International Society of Preventive \& Community Dentistry, vol. 9, no. 5, p. 499, 2019.

[14] S. E. Zange, A. L. Ramos, M. R. De Mendonça, and R. Suguino, "Perceptions of laypersons and orthodontists regarding the buccal corridor in long- and short-face individuals," The Angle Orthodontist, vol. 81, no. 1, pp. 86-90, 2011.

[15] M. Cuoghi, A. Cláudia de Castro Ferreira Conti, R. R. Almeida Pedrin, V. De Miranda Ladewig, and M. A. Cardoso, "Evaluation of facial esthetics in long-faced white Brazilian middle school students," American Journal of Orthodontics and Dentofacial Orthopedics, vol. 155, no. 6, pp. 812-818, 2019.

[16] P. L. E. Filho and J. N. Mucha, "Comparison of two scales for evaluation of smile and dental attractiveness," Dental Press Journal of Orthodontics, vol. 20, no. 2, pp. 42-48, 2015.

[17] A. Guerra, C. Santariello, V. Assi, F. Ballanti, and P. Cozza, "Influence of activation protocol on perceived pain during rapid maxillary expansion," The Angle Orthodontist, vol. 85, no. 6, pp. 1015-1020, 2015.

[18] A. Nota, D. Kim, J. Y. Tang, and H. B. Gladstone, "Quantifying soft tissue loss in facial aging: a study in women using magnetic resonance imaging," Dermatologic Surgery, vol. 39, no. 12, pp. 1895-1902, 2013.

[19] S. Joseph, T. McPhail, and J. Warren, "Image deformation using moving least squares," ACM Transactions on Graphics, vol. 25, no. 3, pp. 533-540, 2006.

[20] J. Ma, J. Zhao, and J. Tian, "Nonrigid image deformation using moving regularized least squares," IEEE Signal Processing Letters, vol. 20, no. 10, pp. 988-991, 2013. 
[21] H. Zhou, "Non-rigid image deformation algorithm based on MRLS-TPS," in Proceedings of the IEEE International Conference on Image Processing (ICIP), IEEE, Beijing, China, September 2017.

[22] J. Ma, J. Zhao, Y. Ma, A. L. Yuille, and Z. Tu, "Robust," IEEE Transactions on Signal Processing, vol. 63, no. 5, pp. 11151129, 2015.

[23] H. Qiu, "Feature guided non-rigid image/surface deformation via moving least squares with manifold regularization," in Proceedings of the IEEE International Conference on Multimedia and Expo (ICME), IEEE, Hong Kong, China, August 2017.

[24] A. Vedaldi and B. Fulkerson, "VLFeat: an open and portable library of computer vision algorithms," in Proceedings of the 18th ACM International Conference on Multimedia, Boston, MA, USA, November 2010.

[25] E. Sauget, "Comparison of occlusal contacts with use of hawley and clear overlay retainers," The Angle Orthodontist, vol. 67 , no. 3, pp. 223-230, 1997.

[26] L. Hichens, "Cost-effectiveness and patient satisfaction: hawley and vacuum-formed retainers," The European Journal of Orthodontics, vol. 29, no. 4, pp. 372-378, 2007.

[27] M. Nomura, "Esthetic preferences of european american, hispanic american, japanese, and african judges for soft-tissue profiles," American Journal of Orthodontics and Dentofacial Orthopedics, vol. 135, no. 4, pp. S87-S95, 2009.

[28] B. J. Schabel, "Subjective vs objective evaluations of smile esthetics," American Journal of Orthodontics and Dentofacial Orthopedics, vol. 135, no. 4, pp. S72-S79, 2009.

[29] K. Hata and K. Arai, "Dimensional analyses of frontal posed smile attractiveness in Japanese female patients," The Angle Orthodontist, vol. 86, no. 1, pp. 127-134, 2016.

[30] H. Ioi, "Anteroposterior lip positions of the most-favored Japanese facial profiles," American Journal of Orthodontics and Dentofacial Orthopedics, vol. 128, no. 2, pp. 206-211, 2005.

[31] T. Shimomura, "Evaluation of well-balanced lip position by Japanese orthodontic patients," American Journal of Orthodontics and Dentofacial Orthopedics, vol. 139, no. 4, pp. e291-e297, 2011. 